PREPARED FOR THE U.S. DEPARTMENT OF ENERGY, UNDER CONTRACT DE-AC02-76CH03073

PPPL-3680

PPPL-3680

UC-70

Transparency of Magnetized Plasma at Cyclotron Frequency

by

G. Shvets and J.S. Wurtele

March 2002

NM|

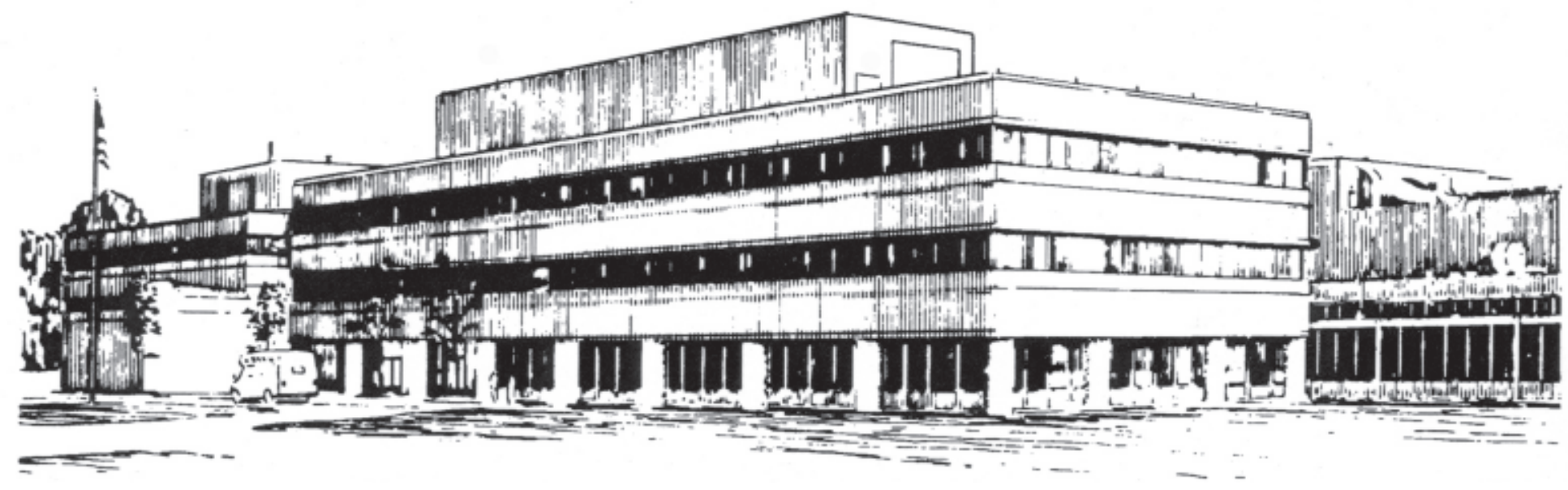

PRINCETON PLASMA PHYSICS LABORATORY PRINCETON UNIVERSITY, PRINCETON, NEW JERSEY 


\section{PPPL Reports Disclaimer}

This report was prepared as an account of work sponsored by an agency of the United States Government. Neither the United States Government nor any agency thereof, nor any of their employees, makes any warranty, express or implied, or assumes any legal liability or responsibility for the accuracy, completeness, or usefulness of any information, apparatus, product, or process disclosed, or represents that its use would not infringe privately owned rights. Reference herein to any specific commercial product, process, or service by trade name, trademark, manufacturer, or otherwise, does not necessarily constitute or imply its endorsement, recommendation, or favoring by the United States Government or any agency thereof. The views and opinions of authors expressed herein do not necessarily state or reflect those of the United States Government or any agency thereof.

\section{Availability}

This report is posted on the U.S. Department of Energy's Princeton Plasma Physics Laboratory Publications and Reports web site in Fiscal Year 2002. The home page for PPPL Reports and Publications is: http://www.pppl.gov/pub_report/

DOE and DOE Contractors can obtain copies of this report from:

U.S. Department of Energy

Office of Scientific and Technical Information

DOE Technical Information Services (DTIS)

P.O. Box 62

Oak Ridge, TN 37831

Telephone: (865) 576-8401

Fax: (865) 576-5728

Email: reports@adonis.osti.gov

This report is available to the general public from:

National Technical Information Service

U.S. Department of Commerce

5285 Port Royal Road

Springfield, VA 22161

Telephone: 1-800-553-6847 or

(703) 605-6000

Fax: (703) 321-8547

Internet: http://www.ntis.gov/ordering.htm 


\title{
Transparency of Magnetized Plasma at Cyclotron Frequency
}

\author{
G. Shvets \\ Princeton Plasma Physics Laboratory, Princeton University, Princeton, NJ 08543 \\ J. S. Wurtele \\ University of California, Berkeley, CA 94720
}

\begin{abstract}
Electromagnetic radiation is strongly absorbed by a magnetized plasma if the radiation frequency equals the cyclotron frequency of plasma electrons. It is demonstrated that absorption can be completely canceled in the presence of a magnetostatic field of an undulator, or a second radiation beam, resulting in plasma transparency at the cyclotron frequency. This effect is reminiscent of the electromagnetically-induced transparency (EIT) of the three-level atomic systems, except that it occurs in a completely classical plasma. Unlike the atomic systems, where are all the excited levels required for EIT exist in each atom, this classical EIT requires the excitation of the non-local plasma oscillation. The complexity of the plasma system results in an index of refraction at the cyclotron frequency that differs from unity. Lagrangian description was used to elucidate the physics and enable numerical simulation of the plasma transparency and control of group and phase velocity. This control naturally leads to applications for electromagnetic pulse compression in the plasma and electron/ion acceleration.
\end{abstract}

PACS: 52.35.Hr, 52.35.Mw, 42.25.Bs, 52.38.Kd 
Electromagnetically induced transparency (EIT) in quantum-mechanical atomic systems is a well understood and thoroughly studied [1] subject. EIT is the basis of several applications, such as slow light [2], information transfer between matter and light [3,4], sound wave generation [5], and even a proposed testing of black-hole physics [6]. Several recent reviews [7] elucidated the quantum mechanical mechanism of EIT, which relies on the destructive interference between several pathways which connect the ground and excited states of the atom. This Letter presents the physics of induced transparency and reduced group velocity in a classical plasma and proposes applications to accelerators and energy compression.

We consider an externally magnetized plasma with $\vec{B}=B_{0} \vec{e}_{z}$ and density $n_{0}$. A righthand polarized electromagnetic wave (which we refer to as the probe) at a frequency $\omega_{1}$ equal to cyclotron frequency $\Omega_{0}=e B_{0} / m c$ cannot propagate in the plasma because it undergoes resonant cyclotron absorption [8]. The cold magnetized plasma dispersion relation $\omega_{1}$ v. s. $k_{1}$ for the right-hand polarized probe, plotted in Fig. 1, is given by $\omega_{1}^{2}=k_{1}^{2} c^{2}+\frac{\omega_{p}^{2} \omega_{1}}{\omega_{1}-\Omega_{0}}$. Both the plasma current and the wavenumber $k_{1}$ become infinite for $\omega_{1} \rightarrow \Omega_{0}$, and a forbidden bandgap exists between $\Omega_{0}$ and $\Omega_{c}=\sqrt{\Omega_{0}^{2} / 4+\omega_{p}^{2}}+\Omega_{0} / 2$, where $\omega_{p}=\left(4 \pi e^{2} n_{0} / m\right)^{1 / 2}$ is the plasma frequency. Transparency can be realized by adding a second intense electromagnetic wave (the pump) with frequency $\omega_{0}=\Omega_{0}-\omega_{p}$. Moreover, if $\omega_{p}=\Omega_{c}$, transparency can be obtained using a magnetostatic undulator with arbitrary wavenumber $k_{0}$.

The mechanism of classical electromagnetically induced transparency is the destructive interference between the electric field of the probe $\left(\vec{E}_{1 \perp}\right)$ and the sidebands of the electric $\left(\vec{E}_{0 \perp}\right)$ and magnetic $\left(\vec{B}_{0 \perp}\right)$ fields of the pump. The sidebands are detuned by the plasma frequency from the pump, and are produced by the collective electron plasma oscillation, with frequency $\omega_{p}$, along the magnetic field. Qualitatively, the total force at the cyclotron frequency experienced by a plasma electron is given by $\vec{F}_{\text {tot }} \approx-e\left(\vec{E}_{1 \perp}+\zeta_{z} \partial_{z} \vec{E}_{0 \perp}+\dot{\zeta}_{z} \vec{e}_{z} \times \vec{B}_{0 \perp}\right)$, where $\zeta_{z}$ is the electron displacement in the plasma wave. In linear theory, with only the probe wave, the resonant $\vec{E}_{1 \perp}$ generates an induced plasma current that dominates the displacement current and prevents wave propagation. With both the pump and probe, if 
the amplitudes and phases of the pump and the plasma wave are properly correlated, the net force can vanish $\left(\vec{F}_{\text {tot }} \sim 0\right)$. Consequently, the plasma current at the cyclotron frequency is small (or even vanishing), and the probe propagates as if in vacuum. Numerical simulation below demonstrates that this correlation is naturally achieved in a collisionless plasma.

We assume two right-hand polarized EM waves propagating along $z$ - direction, with their electric and magnetic fields given by $2 e \vec{E}_{0 \perp} / m c \omega_{0}=a_{\text {pump }} \vec{e}_{+} \exp \left(i \bar{\theta}_{0}\right)+c . \quad c$., $2 e \vec{E}_{1 \perp} / m c \omega_{1}=a_{\text {probe }} \vec{e}_{+} \exp \left(i \vec{\theta}_{1}\right)+c . c$. and $\vec{B}_{0,1 \perp}=\left(c \vec{k}_{0,1} / \omega_{0,1}\right) \times \vec{E}_{0,1 \perp}$, where $\vec{e}_{ \pm}=\vec{e}_{x} \pm i \vec{e}_{y}$ and $\bar{\theta}_{0,1}=k_{0,1} z-\omega_{0,1} t$. The non-relativistic equation of motion of a plasma electron in the combined fields is given by

$$
\frac{d^{2} \vec{x}}{d t^{2}}+\Omega_{0} \vec{v} \times \vec{e}_{z}+\omega_{p}^{2} \zeta_{z} \vec{e}_{z}=-\frac{e}{m} \sum_{m=0,1}\left(\vec{E}_{m \perp}+\frac{\vec{v} \times \vec{B}_{\perp m}}{c}\right),
$$

where $\vec{x} \equiv\left(z_{0}+\zeta_{z}\right) \vec{e}_{z}+\vec{x}_{\perp}$ and $\vec{v}=d \vec{x} / d t \equiv c \vec{\beta}$ are the particle position and velocity. The initial conditions are $\vec{v}=0$ and $\vec{x}=z_{0} \vec{e}_{z}$. The third term on the LHS of Eq. (1) is the restoring force of the ions [9].

Equation (1) was integrated for the case when only a probe field is present and the case with both the pump and the probe. The pump and the probe amplitudes were taken as increasing adiabatically in time, up to their respective peak amplitudes of $a_{0}$ and $a_{1}$, according to

$$
\begin{aligned}
& a_{\text {pump }}=\frac{a_{0}}{2}\left(1+\tanh \left[\left(\Omega_{0} t-160\right) / 40\right]\right), \\
& a_{\text {probe }}=\frac{a_{1}}{2}\left(1+\tanh \left[\left(\Omega_{0} t-320\right) / 40\right]\right) .
\end{aligned}
$$

This enables the pump to turn on first, followed by the probe.

Simulation results for $\omega_{p} / \Omega_{0}=0.3\left(\omega_{0}=0.7 \Omega_{0}\right)$ are shown in Fig. 2. Without the pump, an electron is resonantly driven by the probe as shown in Fig. 2(a). In the plasma, this growth leads to a large electron current and consequent probe absorption (time-averaged, $\left.\vec{E}_{\perp} \cdot \vec{v}_{\perp}<0\right)$. Adding a strong pump with $a_{0}=0.1$ and $k_{0} \approx 0.83 \Omega_{0} / c$ dramatically changes electron motion, as seen in Fig. 2(b). After the pump is turned on but before the turning on of the probe, an electron oscillates in the field of the pump according to the analytic solution 
$\beta_{x 0}=\omega_{0} a_{\text {pump }} /\left(\omega_{0}-\Omega_{0}\right) \sin \left(k_{0} z_{0}-\omega_{0} t\right)$. Switching on the probe does not significantly alter electron motion: $\beta_{x}-\beta_{x 0}$ appears as a barely visible dashed line in Fig. 2(b). Figs. 2(a) and (b) illustrate how the pump suppressed electron response at the cyclotron frequency. This suppression results in the plasma becoming transparent at the cyclotron frequency and allows the propagation of the probe.

The suppression of the linear response to the probe is caused by the excitation of a strong plasma oscillation [shown in Fig. 2(c)], which produces a sideband of the pump at the cyclotron frequency. This sideband, in turn, cancels the electric field of the probe. An approximate analytic formula for the steady-state the plasma oscillation,

$$
\zeta_{0}=\frac{2 a_{\text {probe }}}{k_{0} a_{\text {pump }}} \sin \omega_{p} t
$$

is derived below by requiring that the sideband cancels the probe. It is in good agreement with the simulation, as seen in in Fig. 2(c) where the dashed line shows the difference between the analytic and simulated motion. Simulation results demonstrate the stability of the steady-state values of $\beta_{x}$ and $\zeta_{z}$; these values are naturally reached in a collisionless plasma. Note that the pump has to be switched on prior to the arrival of the probe. In atomic physics, this pulse sequence is well-known, and referred to as "counter-intuitive" [7]. Here it seems intuitive, in that the pump needs to be present so as to suppress the response to the probe.

Generating a high-power pump wave may prove challenging in practice. For example, a dimensionless vector potential of $a_{0}=0.01$ over an area $A=\left(2 \pi c / \omega_{0}\right)^{2}$ requires microwave power of order $3 \mathrm{MW}$. Transparency can, however, also be induced by a helical undulator field. In this circumstance, corresponding to a zero-frequency pump, one requires $\omega_{p}=\Omega_{0}$. We simulated electron motion in the combined field of an undulator, with $a_{0}=0.1$ and $k_{0}=2 \Omega_{0} / c$, and a probe, switched on according to $a_{\text {probe }}=0.5 a_{1}\left(1+\tanh \left[\left(\Omega_{0} t-270\right) / 60\right]\right)$, where $a_{1}=0.01$. Suppression of the electron response at the cyclotron frequency is apparent from Fig. 3(a). The force due to the electric field of the probe is canceled by the $\left(\dot{\zeta}_{z} / c\right) \vec{e}_{z} \times \vec{B}_{0 \perp}$ force which is exerted on a longitudinal plasma wave by the helical magnetic field of the 
undulator. The plasma wave in this example can be used for acceleration of relativistic electrons because its phase velocity is $v_{p h}=\left(\omega_{1}-\omega_{0}\right) /\left(k_{1}-k_{0}\right)=-c$.

The steady-state values of $\beta_{+}=\beta_{-}^{*}=\beta_{x}-i \beta_{y}$ and $\zeta_{z}$ can be analytically obtained by a straightforward linearization of Eq. (1) in the weak probe, $a_{1} \ll a_{0}$, limit.

$$
\begin{aligned}
& \dot{\beta}_{+}+i \Omega_{0} \beta_{+}= \\
& -\left(\omega_{0} a_{0} e^{i \bar{\theta}_{0}}+\omega_{1} a_{1} e^{i \bar{\theta}_{1}}-k_{0} a_{0} \dot{\zeta}_{z} e^{i \bar{\theta}_{0}}-k_{1} a_{1} \dot{\zeta}_{z} e^{i \bar{\theta}_{1}}\right) .
\end{aligned}
$$

Introducing $\theta_{0,1}=k_{0,1} z_{0}-\omega_{0,1} t$ and assuming that $k_{0,1} \zeta_{z}<1$, the exponentials in Eq. (4) are expanded as $e^{i \bar{\theta}_{0,1}} \approx e^{i \theta_{0,1}}\left(1+i k_{0,1} \zeta_{z}\right)$, yielding

$$
\begin{aligned}
& \dot{\beta}_{+}+i \Omega_{0} \beta_{+}=-\omega_{0} a_{0} e^{i \theta_{0}}\left(1+i k_{0} \zeta_{z}-k_{0} \dot{\zeta}_{z} / \omega_{0}\right) \\
& -\omega_{1} a_{1} e^{i \theta_{1}}\left(1+i k_{1} \zeta_{z}-k_{1} \dot{\zeta}_{z} / \omega_{1}\right) .
\end{aligned}
$$

The longitudinal equation of motion is given by

$$
\ddot{\zeta}_{z}+\omega_{p}^{2} \zeta_{z} \approx-\frac{e}{m c}\left(\vec{v}_{\perp} \times \vec{B}_{\perp}+\zeta_{z} \vec{v}_{\perp} \times \frac{\partial \vec{B}_{\perp}}{\partial z}\right)
$$

where $\vec{B}_{\perp}(z, t)$ was expanded as $\vec{B}_{\perp}\left(z_{0}+\zeta_{z}\right) \approx \vec{B}_{\perp}\left(z_{0}\right)+\zeta_{z} \partial_{z_{0}} \vec{B}_{\perp}\left(z_{0}\right)$ to first order in $\zeta_{z}$. Expression $\vec{B}_{\perp}$ in terms of amplitudes and phases yields

$$
\begin{aligned}
& \ddot{\zeta}_{z}+\omega_{p}^{2} \zeta_{z}=-\frac{c^{2}}{2}\left(k_{0} a_{0} \beta_{-} e^{i \theta_{0}}+k_{1} a_{1} \beta_{-} e^{i \theta_{1}}-\right. \\
& \left.i k_{0}^{2} \zeta_{z} \beta_{-} a_{0} e^{i \theta_{0}}-i k_{1}^{2} \zeta_{z} a_{1} e^{i \theta_{1}}\right)+c . c .
\end{aligned}
$$

The last term on the RHS of Eq. (6) will be dropped because it is proportional to the product of two small quantities, $\zeta_{z}$ and $a_{1}$. Note that, unlike the transverse velocity $\beta_{+}$which is excited directly by each of the two lasers according to Eq. (5), plasma waves are excited only in the presence of two lasers (including the possibility of one having zero-frequency) via the beatwave mechanisms.

The physical reason for transparency in the plasma is the strong coupling between the longitudinal and transverse degrees of freedom of the plasma electrons. The steady-state solution of Eq. (6) $\zeta_{z}=0.5 \tilde{\zeta} \exp i(\Delta k z-\Delta \omega t)+c . c .$, where $\Delta \omega=\omega_{1}-\omega_{0}$ and $\Delta k=$ 
$k_{1}-k_{0}$, is substituted into the transverse equation of motion (5). Retaining terms with $\exp -i \omega_{0} t$ and $\exp -i \omega_{1} t$ dependence results in

$$
\beta_{+}=-\frac{i \omega_{0} a_{0}}{\omega_{0}-\Omega_{0}} e^{i \theta_{0}}-\frac{i \omega_{1}}{\omega_{1}-\Omega_{0}}\left(a_{1}+\frac{i k_{0} \tilde{\zeta}}{2} a_{0}\right) e^{i \theta_{1}}
$$

Applying Eq. (7) to case of $\omega_{1}=\Omega_{0}$ and $\Delta \omega=\omega_{p}$ yields the steady-state amplitude of the plasma wave given by Eq. (3).

The general case of $\omega_{1} \neq \Omega_{0}$ is handled by inserting $\beta_{+}$and $\beta_{-}$into Eq. (6) to arrive at

$$
\begin{aligned}
& \left(\omega_{p}^{2}-\Delta \omega^{2}\right) \tilde{\zeta}=i c^{2}\left[\frac{k_{0} a_{0}^{*} \omega_{1}}{\omega_{1}-\Omega_{0}}\left(a_{1}+i k_{0} \tilde{\zeta} a_{0} / 2\right)-\right. \\
& \left.\frac{k_{1} a_{1} \omega_{0}}{\omega_{0}-\Omega_{0}} a_{0}^{*}-i \frac{k_{0}^{2} \tilde{\zeta} \omega_{0}}{\omega_{0}-\Omega_{0}}\left|a_{0}\right|^{2}\right],
\end{aligned}
$$

where $\theta_{1}-\theta_{0}=\left(k_{1}-k_{0}\right) z_{0}-\Delta \omega t$. Equation (8) is then solved for $\tilde{\zeta}$. Substitution of this expression for $\tilde{\zeta}$ into Eq. (7) yields the steady-state perpendicular velocity:

$$
\begin{aligned}
& \beta_{+s}=-\frac{i \omega_{0} a_{0}}{\omega_{0}-\Omega_{0}} e^{i \theta_{0}}-i \frac{\omega_{1} a_{1}}{\omega_{0}-\Omega_{0}} e^{i \theta_{1}} \times \\
& \times \frac{c^{2} k_{0}^{2} \omega_{0}\left|a_{0}\right|^{2}\left(k_{1} / k_{0}-2\right)+2\left(\omega_{p}^{2}-\Delta \omega^{2}\right)\left(\omega_{0}-\Omega_{0}\right)}{c^{2} k_{0}^{2}\left|a_{0}\right|^{2} \omega_{1}+2\left(\omega_{p}^{2}-\Delta \omega^{2}\right)\left(\omega_{1}-\Omega_{0}\right)},
\end{aligned}
$$

where we have neglected terms proportional to the product of the laser detuning from resonance, $\delta \Omega=\omega_{1}-\Omega_{0}$, and the pump intensity $a_{0}^{2}$. Qualitatively, the influence of the pump is strong only close to the cyclotron resonance, and is negligible far from $\omega_{1}=\Omega_{0}$. From Eq. (9), plasma is resonantly driven when the denominator $D=2\left(\omega_{p}^{2}-\Delta \omega^{2}\right)\left(\omega_{1}-\Omega_{0}\right)+c^{2} k_{0}^{2}\left|a_{0}\right|^{2} \omega_{1}$ vanishes. Close to cyclotron resonance, $D \approx 4 \omega_{p}\left(\Omega_{R}^{2}-\delta \Omega^{2}\right)$, where $\Omega_{R}=c k_{0} a_{0}\left(\Omega_{0} / 4 \omega_{p}\right)^{1 / 2}$ is the effective Rabi frequency. Hence, the pump field shifts plasma resonances from $\omega_{1}=\Omega_{0}$ to $\omega_{1}=\Omega_{0} \pm \Omega_{R}$

The fluid velocity component synchronous with the probe (i.e., proportional to $\exp i \theta_{1}$ ) is $b_{+} \approx \beta_{+s}-\partial_{z}\left(\zeta_{z} \beta_{+s}\right)=\beta_{+}-i k_{1} \zeta_{z} \beta_{+}$Explicitly, using the results of the last paragraph, $b_{+}=i a_{1} \omega_{1}\left(\delta \Omega+\delta \Omega_{0}\right) /\left(\Omega_{R}^{2}-\delta \Omega^{2}\right)$ where $\delta \Omega_{0}\left(k_{1}\right)=\left(2 \Omega_{R}^{2} \omega_{0} / \omega_{p} \Omega_{0}\right)\left(k_{1} / k_{0}-1\right)$. The expression for $b_{+}$can now be used to calculate the current in the plasma that is synchronous with the probe field. 
The desired dispersion relation for induced transparency in a magnetized plasma is derived from the wave equation for the probe, $-\left(c^{2} \partial_{z}^{2}-\partial_{t}^{2}\right) \vec{E}=4 \pi \partial_{t} \vec{J}=-4 \pi i c \omega_{1} e n_{0} b_{+}+c . c$. . One finds

$$
\omega_{1}^{2}=c^{2} k_{1}^{2}-\omega_{p}^{2} \omega_{1} \frac{\delta \Omega+\delta \Omega_{0}\left(k_{1}\right)}{\Omega_{R}^{2}-(\delta \Omega)^{2}},
$$

where it was assumed that the frequency of the pump is fixed at $\omega_{0}=\Omega_{0}-\omega_{p}$. Complete transparency $\left(\omega_{1}^{2}=k_{1}^{2} c^{2}\right)$ is achieved at $\omega_{1}=\Omega_{0}-\delta \bar{\Omega}_{0}$, where $\delta \bar{\Omega}_{0} \approx\left(2 \omega_{0} \Omega_{R}^{2} / \omega_{p} \Omega_{0}\right)\left(\Omega_{0} / k_{0} c-\right.$ 1). Note that this frequency shift is in general very small in the most interesting regime of $\Omega_{R} \ll \omega_{p}:\left|\delta \Omega_{0}\right|<4 \Omega_{R}^{2} / \omega_{p} \ll \Omega_{R}$, and can be even smaller near cyclotron resonance when the pump and probe co-propagate. Equation (10) reduces to the dispersion relation for a single probe in a magnetized plasma when the detuning is large, $(\delta \Omega)^{2} \gg \Omega_{R}^{2}$. As expected, the influence of the pump on the response of the plasma to the probe is significant only in the vicinity of $\delta \Omega=0$.

Note that index of refraction is not identically equal to unity at the cyclotron resonance. This is different from the quantum-mechanical result for a three-level system [10], where $\omega_{1}=k_{1} c$ on resonance. We speculate that this difference occurs because of multiple Landau levels $E_{n}=n \hbar \Omega_{0}$ and corresponding Raman-shifted levels $E_{R n}=E_{n}+\hbar \omega_{p}$ which participate in the classical EIT.

The modified dispersion relation given by Eq. (10) is plotted in Fig. (4), for the same plasma parameters as in Fig. (1) and a co-propagating pump with $\Omega_{R}=0.5 \omega_{p}$. The flat band between the $\Omega_{0} \pm \Omega_{R}$ resonant frequencies is a novel feature which is not present without the pump (compare with Fig. (1)). The width of this transparency band, which scales as $\Omega_{R} \propto$ $a_{0}$, can become very narrow for low pump amplitude. The corresponding "group velocity" (as understood in the strictly geometrical sense explained below) $v_{g}=\partial \omega_{1} / \partial k_{1} \approx 2 c \Omega_{R}^{2} / \omega_{p}^{2}$ can also be made arbitrarily small. This slowly propagating wavepacket of electromagnetic waves is a classical analog of the "slow light" in atomic systems [2].

Qualitatively, the spectacular slowing down of electromagnetic waves in the EIT plasma can be understood by considering the entrance of a probe beam of duration $L_{0}$ into the 
plasma. In steady-state inside the plasma, the "slow light" wavepacket of length $L_{f}$ consists of the transversely polarized field of the probe $\left|\vec{E}_{1}\right|=\left|\vec{B}_{1}\right|=a_{1} m c \omega_{1} / e$ and the longitudinal electric field of the plasma wave $E_{z}=4 \pi e n_{0}\left(2 a_{1} / k_{0} a_{0}\right)$. As the pulse enters the plasma, it loses photons to the pump at the same rate as new plasmons are created (according to the Manley-Rowe relations). The classical photon density of a field with frequency $\omega$ is proportional to the action density $\propto U / \omega$, where $U$ is the energy density. From this one can calculate that the ratio of the plasmon to photon density inside the "slow light" pulse,

$$
\frac{U_{\text {plas }} / \omega_{p}}{U_{\text {phot }} / \omega_{1}}=\frac{\Omega_{0}}{\omega_{p}} \frac{E_{z}^{2}}{2 E_{1}^{2}}=\frac{\omega_{p}^{2}}{2 \Omega_{R}^{2}},
$$

is much greater than unity if $\Omega_{R} \ll \omega_{p}$. Thus, most of photons of the original pulse are transferred to the plasma wave. Since the index of refraction remains close to unity, the photon energy density in the pulse remains roughly constant. Therefore, the loss of photons is due to the spatial shortening of the pulse from $L_{0}$ to $L_{f}=L_{0} \times\left(2 \Omega_{R}^{2} / \omega_{p}^{2}\right)$. Because temporal pulse duration does not change, we recover the previously calculated $v_{g} / c=2 \Omega_{R}^{2} / \omega_{p}^{2}$. It is precisely in this geometric sense of $v_{g} / c=L_{f} / L_{0}$ that the group velocity of the slow light is interpreted. $v_{g}$ is not related to the speed of individual photons since their number is not conserved during the pulse transition into the plasma. In the case of a static undulator all the energy is transfered to the plasma and compressed by a factor $v_{g} / c$, resulting in a dramatic increase of the energy density.

One interesting application of EIT in magnetized plasma is ion acceleration. While laserplasma accelerators of electrons [11] have long been considered as a long-term alternative to conventional rf cavity-based linacs, the field of plasma-based ion accelerators is still in its infancy [12]. Present concepts revolve around ions generated at low energies when ultraintense lasers hit thin foils. The plasma system studies here enables one to conceive of an entirely different scheme-a short-pulse ion accelerator consisting of a "slow light" pulse in a plasma with approximately equal group and phase velocities. Acceleration is accomplished by the longitudinal electric field of the plasma wave. Counter-propagating geometry is chosen to match the phase and group velocities because $v_{\mathrm{ph}}=\omega_{p} /\left|k_{0}\right|+k_{1} \approx 0.5 c \omega_{p} / \Omega_{0}$. 
Matching $v_{\mathrm{ph}}=v_{g}$ yields $a_{0} \approx \omega_{p}^{2} / \Omega_{0}^{2} \ll 1$. Other accelerator concepts which rely on the ponderomotive force appear attractive because the ponderomotive force, which scales as the gradient of the energy density $E_{z}^{2} / L_{f} \propto\left(\omega_{p} / \Omega_{0}\right) U_{0} / v_{g}^{2}$, increases rapidly with decreasing group velocity of the probe.

This work was supported by the DOE Division of High Energy Physics and the Presidential Early Career Award for Scientists and Engineers. 


\section{REFERENCES}

[1] K. J. Boller, A. Imamoglu, and S. E. Harris, Phys. Rev. Lett. 66, 2593 (1991); S. E. Harris, Phys. Rev. Lett. 70, 552 (1993).

[2] L. V. Hau et. al., Nature 397, 594 (2001).

[3] M. Fleischhauer, S. F. Yelin, and M. D. Lukin, Opt. Commun. 179, 395 (2000).

[4] M. D. Lukin, S. F. Yelin, and M. Fleischhauer, Phys. Rev. Lett. 84, 4232 (2000).

[5] A. B. Matsko, Y. V. Rostovtsev, M. Fleischhauer, and M. O. Scully, Phys. Rev. Lett. 86, 2006 (2001).

[6] U. Leonhardt and P. Piwnicki, Phys. Rev. Lett. 84, 822 (2000).

[7] S. E. Harris, Physics Today 7, 36 (1997); J. P. Marangos, Journ. Modern Optics 45, 471 (1998); A. B. Matsko et. al., Advances in Atomic, Molecular, and Optical Physics 46, 191 (2001).

[8] N. A. Krall and A. W. Trivelpiece, Principles of Plasma Physics, Chapt. 4 (McGrawHill, New York, 1973).

[9] J. Dawson, Phys. Rev. 113, 383 (1959).

[10] M. O. Scully, Phys. Rev. Lett. 67, 1855 (1991); M. Fleischhauer, C. H. Keitel, and M. O. Scully, Phys. Rev. A 46, 1468 (1992).

[11] T. Tajima and J. M. Dawson, Phys. Rev. Lett. 43, 267 (1979).

[12] R. A. Snavely et. al., Phys. Rev. Lett. 85, 2945 (2000). 


\section{FIGURES}

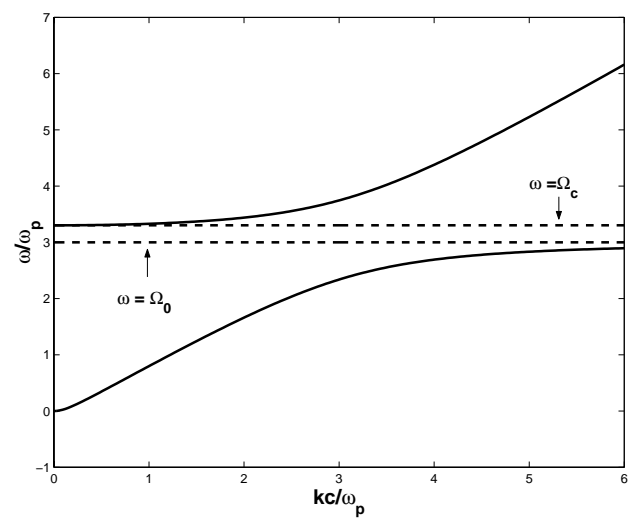

FIG. 1. Dispersion curve for a right-hand polarized wave propagating along a magnetic field. A forbidden gap exists between the cyclotron frequency $\Omega_{0}=e B_{0} / m c$ and the cutoff frequency $\Omega_{c}=\Omega_{0} / 2+\sqrt{\Omega_{0}^{2} / 4+\omega_{p}^{2}}$
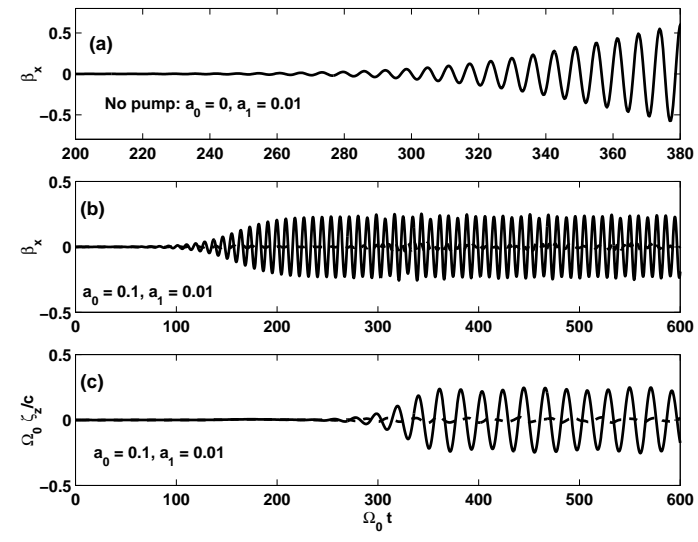

FIG. 2. Numerical simulation of single particle motion in the combined field of two EM waves with $\left(\omega_{1}=\Omega_{0}, k_{1}=\omega_{1} / c\right)$ and $\left(\omega_{0}=\Omega_{0}-\omega_{p}, k_{0} \approx 0.83 \Omega_{0} / c\right)$. Both the pump and the probe are slowly turned on according to Eq. (2). (a) Without the pump an electron is resonantly driven by probe: $\beta_{x}$ growth indefinitely; (b) With the pump, electron motion is almost unaffected by the probe. Solid line - total $\beta_{x}$; barely visible dashed line $-\left(\beta_{x}-\beta_{x 0}\right)$, where $\beta_{x 0}=\omega_{0} a_{\text {pump }} /\left(\omega_{0}-\Omega_{0}\right) \sin \left(k_{0} z_{0}-\omega_{0} t\right)$ is the analytic result when only the pump is present. (c) Solid line: the longitudinal displacement $\Omega_{0} \zeta_{z} / c$; dashed line: $\Omega_{0}\left(\zeta_{z}-\zeta_{0}\right) / c$, where $\zeta_{0}=2 a_{\text {pump }} / a_{\text {probe }} \sin \omega_{p} t$ from Eq. (3) 

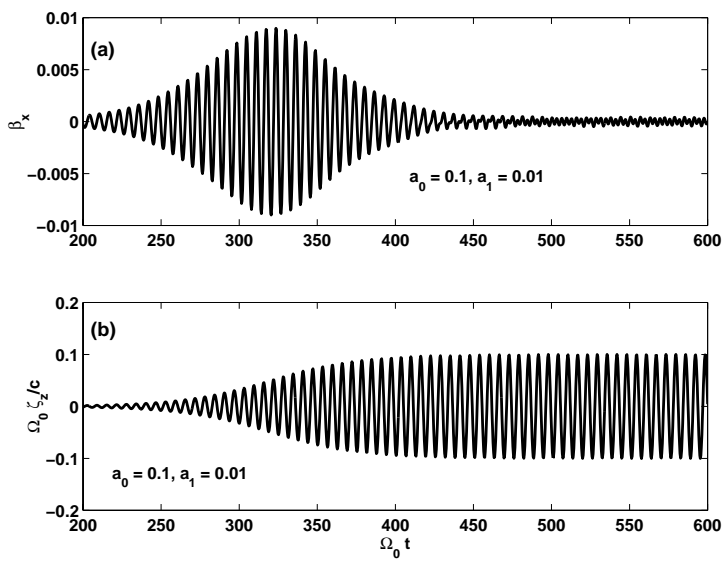

FIG. 3. The same parameters as Fig. 2, except $\omega_{p}=\Omega_{0}, \omega_{0}=0, k_{0}=2 \Omega_{0} / c$ (the static helical undulator is on continuously). (a) The transverse velocity $\beta_{x}$ and (b) the longitudinal displacement $\Omega_{0} \zeta_{z} / c$ during and after the turn-on of the probe.

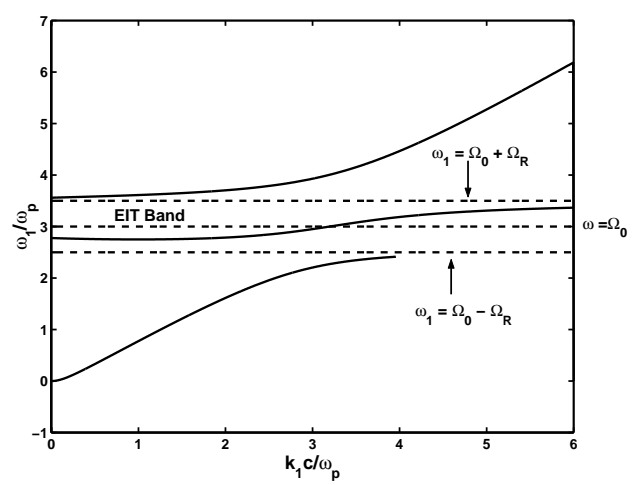

FIG. 4. EIT dispersion curve, $\Omega_{0} / \omega_{p}=3$ and $\Omega_{R} / \omega_{p}=1 / 2$. Flat band above $\Omega_{0}-\Omega_{R}$ up to $\Omega_{0}+\Omega_{R}$ labeled "EIT Band" corresponds to "slow light" and appears only in the presence of a pump. 


\section{External Distribution}

Plasma Research Laboratory, Australian National University, Australia

Professor I.R. J ones, Flinders University, Australia

Professor J oão Canalle, Instituto de Fisica DEQ/IF - UERJ , Brazil

Mr. Gerson O. Ludwig, Instituto Nacional de Pesquisas, Brazil

Dr. P.H. Sakanaka, Instituto Fisica, Brazil

The Librarian, Culham Laboratory, England

Library, R61, Rutherford Appleton Laboratory, England

Mrs. S.A. Hutchinson, JET Library, England

Professor M.N. Bussac, Ecole Polytechnique, France

Librarian, Max-Planck-Institut für Plasmaphysik, Germany

J olan Moldvai, Reports Library, MTA KFKI-ATKI, Hungary

Dr. P. Kaw, Institute for Plasma Research, India

Ms. P.J . Pathak, Librarian, Insitute for Plasma Research, India

Ms. Clelia De Palo, Associazione EURATOM-ENEA, I taly

Dr. G. Grosso, Instituto di Fisica del Plasma, Italy

Librarian, Naka Fusion Research Establishment, J AERI, J apan

Library, Plasma Physics Laboratory, Kyoto University, J apan

Research Information Center, National Institute for Fusion Science, J apan

Dr. O. Mitarai, Kyushu Tokai University, J apan

Library, Academia Sinica, Institute of Plasma Physics, People's Republic of China

Shih-Tung Tsai, Institute of Physics, Chinese Academy of Sciences, People's Republic of China

Dr. S. Mirnov, TRINITI, Troitsk, Russian Federation, Russia

Dr. V.S. Strelkov, Kurchatov Institute, Russian Federation, Russia

Professor Peter Lukac, Katedra Fyziky Plazmy MFF UK, Mlynska dolina F-2, Komenskeho Univerzita, SK-842 15 Bratislava, Slovakia

Dr. G.S. Lee, Korea Basic Science Institute, South Korea

Mr. Dennis Bruggink, Fusion Library, University of Wisconsin, USA

Institute for Plasma Research, University of Maryland, USA

Librarian, Fusion Energy Division, Oak Ridge National Laboratory, USA

Librarian, Institute of Fusion Studies, University of Texas, USA

Librarian, Magnetic Fusion Program, Lawrence Livermore National Laboratory, USA

Library, General Atomics, USA

Plasma Physics Group, Fusion Energy Research Program, University of California at San Diego, USA

Plasma Physics Library, Columbia University, USA

Alkesh Punjabi, Center for Fusion Research and Training, Hampton University, USA

Dr. W.M. Stacey, Fusion Research Center, Georgia Institute of Technology, USA

Dr. J ohn Willis, U.S. Department of Energy, Office of Fusion Energy Sciences, USA

Mr. Paul H. Wright, Indianapolis, Indiana, USA 
The Princeton Plasma Physics Laboratory is operated by Princeton University under contract with the U.S. Department of Energy.

\author{
Information Services \\ Princeton Plasma Physics Laboratory \\ P.O. Box 451 \\ Princeton, NJ 08543
}

Phone: 609-243-2750

Fax: 609-243-2751

e-mail: pppl_info@pppl.gov

Internet Address: http://www.pppl.gov 